\title{
Mechanosorptive creep in nanocellulose materials
}

\author{
Stefan B Lindström, Erdem Karabulut, Artem Kulachenko, \\ Houssine Sehaqui and Lars Wågberg
}

\section{Linköping University Post Print}

N.B.: When citing this work, cite the original article.

The original publication is available at www.springerlink.com:

Stefan B Lindström, Erdem Karabulut, Artem Kulachenko, Houssine Sehaqui and Lars Wågberg, Mechanosorptive creep in nanocellulose materials, 2012, Cellulose (London), (19), 3, 809-819.

http://dx.doi.org/10.1007/s10570-012-9665-9

Copyright: Springer Verlag (Germany)

http://www.springerlink.com/?MUD=MP

Postprint available at: Linköping University Electronic Press

http://urn.kb.se/resolve?urn=urn:nbn:se:liu:diva-77001 


\title{
Mechanosorptive creep in nanocellulose materials
}

\author{
S.B. Lindström ${ }^{1 *}$ E. Karabulut ${ }^{2}$, A. Kulachenko ${ }^{3}$, \\ H. Sehaqui ${ }^{2}$ and L. Wågberg ${ }^{2}$ \\ ${ }^{1}$ Mechanics, Department of Management and Engineering, The Institute of \\ Technology, Linköping University, SE-581 83 Linköping, Sweden. \\ ${ }^{2}$ Department of Fiber and Polymer Technology, KTH Royal Institute of \\ Technology, Teknikringen 56-58, SE-100 44 Stockholm, Sweden. \\ ${ }^{3}$ Department of Solid Mechanics, KTH Royal Institute of Technology, \\ Osquars backe 1, SE-100 44 Stockholm, Sweden.
}

June 25, 2012

\begin{abstract}
The creep behavior of nanocellulose films and aerogels are studied in a dynamic moisture environment, which is crucial to their performance in packaging applications. For these materials, the creep rate under cyclic humidity conditions exceeds any constant humidity creep rate within the cycling range, a phenomenon known as mechanosorptive creep. By varying the sample thickness and relative humidity ramp rate, it is shown that mechanosorptive creep is not significantly affected by the through-thickness moisture gradient. It is also shown that cellulose nanofibril aerogels with high porosity display the same accelerated creep as films. Microstructures larger than the fibril diameter thus appear to be of secondary importance to mechanosorptive creep in nanocellulose materials, suggesting that the governing mechanism is found between molecular scales and the length-scales of the fibril diameter.
\end{abstract}

\section{Introduction}

The remarkable specific stiffness, strength and flexibility of wood and plant fibers originate from the composite structure of high aspect ratio cellulose nanofibrils in the fiber wall (Wågberg and Annergren, 1997; Forgacs and Mason, 1958). These nanofibrils, with a diameter as small as $4 \mathrm{~nm}$ (Fall et al, 2011), can be liberated using chemical or enzymatic treatment with subsequent mechanical disintegration (Saito et al, 2007; Henriksson et al, 2007).

\footnotetext{
${ }^{*}$ Corresponding author. e-mail: stefan.lindstroem@gmail.com
} 
The resulting NanoFibrillated Cellulose (NFC) is a renewable, low-cost component in many advanced nanostructured materials, including nanopapers (Henriksson et al, 2008), foams (Aulin et al, 2010), aerogels (Sehaqui et al, 2011) and biocomposites (Capadona et al, 2009; Yousefi et al, 2011). NFC films and foams have potential applications in the packaging industry as sustainable alternatives to petroleum-based solutions. However, cellulosebased materials are hygroscopic and moisture has an adverse effect on their stiffness and strength. Understanding and controlling the mechanical properties of NFC materials in a dynamic moisture environment is thus crucial for enabling these promising materials to become truly pervasive.

Under a sustained loading, typical for packaging applications, the deformation rate of many hygroscopic materials has a strong dependence on fluctuations in the ambient relative humidity $(\mathrm{RH})$. Wood (Armstrong and Kingston, 1960; Armstrong and Christensen, 1961), paper (Byrd, 1972), individual wood fibers (Olsson et al, 2007), fiberboard (Urbanik, 1995), wool (Mackay and Dowes, 1959) and Kevlar (Habeger et al, 2001) are known examples. This phenomenon is known as mechanosorptive creep or accelerated creep. Since the phenomenon threatens the integrity of hygroscopic material structures under a constant loading, and particularly shortens the storage-life of packaging boxes (Leake and Wojcik, 1993), the phenomenon is in itself of great practical and economic importance. Yet, there is no generally accepted theory for its primary mechanism (Habeger and Coffin, 2000). It has been proposed that mechanosorptive creep is caused by enhanced compliance due to moisture transport (Gibson, 1965; Navi et al, 2002) or moisture rate-of-change (Ranta-Maunus, 1975), to a physical de-aging mechanism (Padanyi, 1993) or to material-specific mechanisms. In the case of paper, the generation of dislocations (Söremark and Fellers, 1989) and heterogeneity-driven creep (Habeger and Coffin, 2000) including anisotropic hygroexpansion of fibers (Söremark and Fellers, 1989; Söremark et al, 1993; Haslach, 1994; Alfthan and Gudmundson, 2005) have been suggested. All of these hypotheses assert that mechanosorptive creep is a local phenomenon. It has also been suggested that there is a contribution to mechanosorptive creep from a nonlocal mechanism in that the through-thickness moisture gradient during sorption and desorption induces a macroscopic stress gradient, which enhances the creep rate (Habeger and Coffin, 2000; Habeger et al, 2001). The hypotheses mentioned above are all plausible and each mechanism may contribute to the mechanosorptive creep to some extent. However, none of the aforementioned theories have become generally accepted as the primary mechanism for mechanosorptive creep. This demonstrates a need for more simple model systems and systematic experimental investigations to test the relative importance of the existing hypotheses.

In this work, we study the mechanosorptive creep of solvent-cast cellulose nanofibril films (Fig. 1a) and high-porosity cellulose nanofibril aerogels (Fig. 1b) in a cyclic RH environment. We emphasize that the scope of the 


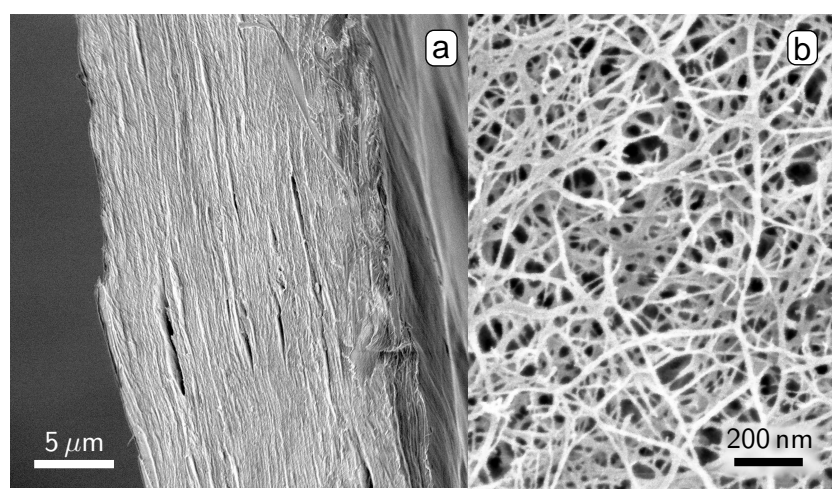

Figure 1: (a) SEM image of the cross-section of a solvent-cast cellulose nanofibril film with basis weight $b=21.4 \mathrm{~g} / \mathrm{m}^{2}$. The exposed cross-section is prepared by manually tearing the film. (b) SEM image of a cellulose nanofibril aerogel.

present investigation is limited to the creep response in tension; the creep in compression does not exhibit the same strain-dependence as the creep in tension, and can be regarded as a separate phenomenon in this respect. Films made from NFC differ from other fibrous cellulosic materials. The constituent fibrils lack the chemical heterogeneity and internal hierarchical structures typical to plant fibers (Fahlén and Salmén, 2002). Investigating the creep in cellulose nanofibril films could thus reveal whether features particular to macroscopic plant fibers, such as anisotropic swelling (Söremark and Fellers, 1989; Söremark et al, 1993; Haslach, 1994), are required for mechanosorptive creep to occur. Moreover, by varying film thickness and $\mathrm{RH}$ ramp rate, the moisture rate-of-change and the through-thickness moisture gradient decouple, so that their respective effects on mechanosorptive creep can be investigated. This addresses the question of locality of mechanosorptive creep. Finally, the effects of microstructures larger than the fibril diameter are investigated by comparing the creep rates of NFC-based films and aerogels. The aerogels are highly porous cellulose nanofibril networks, so their microstructures are much less constrained than those of the films. This should relieve some of the heterogeneity-induced stresses at length-scales larger than the fibril diameter. Investigating the mechanosorptive creep of aerogels will thus indicate whether heterogeneity-induced stress concentrations play an important role, as previously proposed (Habeger and Coffin, 2000).

After a short description of the preparation of materials used and experimental techniques, we present experimental data on the creep of NFC films under constant moisture and in a cyclic humidity environment, respectively. We investigate theoretically and experimentally the dynamics of the through-thickness moisture profile during nonideal RH steps. This detailed 
Table 1: Properties of the solvent-cast cellulose nanofibril films and aerogels. Basis weight $b$ and film thickness $h$ are measured, while density $\rho=b / h$ and volume fraction $\phi=\rho / \rho_{\mathrm{c}}$ are computed. The density of one-chain, triclinic crystal structure cellulose is $\rho_{\mathrm{c}}=1.582 \mathrm{~kg} / \mathrm{L}$ (Sugiyama et al, 1991). The stiffness $E_{80 \%}$ is measured at $80 \% \mathrm{RH}$. The water diffusivity $D$ is obtained using a fitting procedure (see Sec. 4). The material labeled ' $F x$ ' was investigated by Minelli et al (2010).

\begin{tabular}{lccccccc}
\hline ID & Material & $\begin{array}{c}b \\
{\left[\mathrm{~g} / \mathrm{m}^{2}\right]}\end{array}$ & $\begin{array}{c}h \\
{[\mu \mathrm{m}]}\end{array}$ & $\begin{array}{c}\rho \\
{[\mathrm{kg} / \mathrm{L}]}\end{array}$ & $\begin{array}{c}\phi \\
{[\%]}\end{array}$ & $\begin{array}{c}E_{80 \%} \\
{[\mathrm{GPa}]}\end{array}$ & $\begin{array}{c}D \\
{\left[\mu \mathrm{m}^{2} / \mathrm{s}\right]}\end{array}$ \\
\hline F1 & film & 17.0 & $11.3 \pm 0.2$ & 1.51 & 95 & 5.8 & 0.16 \\
F2 & 2 & 21.4 & $14.7 \pm 2.6$ & 1.46 & 92 & 4.8 & 2 \\
F3 & 2 & 30.0 & $19.8 \pm 0.4$ & 1.51 & 95 & 7.5 & 2 \\
A1 & aerogel & 26.7 & 170 & 0.157 & 10 & 0.16 & 43 \\
A2 & 2 & 53.1 & 150 & 0.354 & 22 & 0.56 & 15 \\
Fx & film & 22 & 20 & 1.10 & 70 & - & 0.68 \\
\hline
\end{tabular}

knowledge of the moisture dynamics is used to interpret creep experiments with NFC films of different thicknesses, and to address the question of locality of mechanosorptive creep. Finally, we compare the mechanosorptive creep of NFC films and NFC aerogels to elucidate the importance of heterogeneity-induced stress concentrations during sorption and desorption.

\section{Materials and methods}

Cellulose nanofibrils are prepared from softwood sulphite pulp fibers using 2,2,6,6-tetramethylpiperidin-1-yloxyl (TEMPO)-mediated oxidation (Saito et al, 2007). Films are cast from a $3.0 \mathrm{~g} / \mathrm{L}$ aqueous dispersion of cellulose nanofibrils in deionized water, which is sonicated, poured into a Petri dish and left to dry at room temperature. The amount is varied to produce films of area density in the range $17 \mathrm{~g} / \mathrm{m}^{2}$ to $30 \mathrm{~g} / \mathrm{m}^{2}$. To measure the film thickness, pieces of each film are notched and torn, and the cross-section exposed by the tear (Fig. 1a) is imaged in a field emission scanning electron microscope (Hitachi S-4800, Spectral Solutions, Sweden). The aerogels (Fig. 1b) are prepared using the t-butanol solvent exchange method (Sehaqui et al, 2011). Their constituent NFC fibrils are made from softwood dissolving pulp (Domsjö Fabriker AB, Sweden) which is enzymatically treated and disintegrated as previously described (Henriksson et al, 2007). A micrometer screw gauge is used to measure the thickness of the aerogels. Measured film and aerogel properties are reported in Table 1. Minelli et al (2010), in one instance, used the same NFC stock for film preparation as we use in this paper, for the purpose of measuring transport properties of NFC. Their films were 
cast from a $6.7 \mathrm{~g} / \mathrm{L}$ suspension and dried at an elevated temperature $\left(45^{\circ} \mathrm{C}\right)$. The material properties of their film is included in Table 1 with label ' $F x$ ', for comparison.

The PerkinElmer DMA7e ${ }^{\mathrm{TM}}$ dynamic mechanical analyzer is used for mechanical testing. A dynamic force measurement procedure is used to determine the stiffness at $80 \% \mathrm{RH}$ (Table 1). Because the time-scale of moisture equilibration by diffusion of water vapor into the sample is proportional to the thickness squared, it is essential to use as thin films as possible to maintain a reasonably short experimental time. When these thin films are mounted in the DMA, they are easily damaged or misaligned. Therefore, to increase the bending rigidity and the critical buckling force, a corrugated geometry is created by clamping the film between blocks with sinusoidal surface undulations (Figs. 2ab). This clamping makes the handling of the films easier, but it also introduces prestresses in the loading direction as well as the cross direction. These prestresses are quantified using Finite Element Analysis (FEA) of a quarter of a clamped but otherwise unloaded sample (Figs. 2cd). Linear elasticity with large deflections is used in the analyses. The maximum stress level in the loading direction is about $6 \mathrm{MPa}$ for the $\mathrm{F} 2$ sample. The average absolute stress is much lower, and relaxation during conditioning is likely to reduce the stresses due to the corrugation further. Creep experiments are carried out at about $60 \mathrm{MPa}$ to ensure that effects of the corrugation are negligible.

Humidified air is generated by an RH-200 relative humidity generator (VTI, Hialeah, FL) coupled through a mixing tank of volume $V$ to the sample chamber. A generated step change in the $\mathrm{RH}$ from $H_{0}$ to $H_{1}$ thus produces a relative humidity $H_{\mathrm{s}}(t)=H_{0}+\left(H_{1}-H_{0}\right)\left[1-e^{-q t / V}\right]$ flowing into the sample chamber, where the gas flow rate $q=0.33 \mathrm{~L} / \mathrm{min}$ is kept constant through all experiments. The sample chamber itself has a finite volume of $25 \mathrm{~mL}$, so that the local relative humidity $H(t)$ at the surface of the sample is modulated by complicated flow dynamics, including mixing at the inlet pipe of the chamber as well as boundary layer diffusion near the surfaces of the sample. These dynamics are modeled as a time delay, so that

$$
H(t)=H_{0}+\left(H_{1}-H_{0}\right)\left[1-e^{-k t}\right]
$$

where $k=q / V+k_{\mathrm{m}}$. Here, $k_{\mathrm{m}}^{-1}$ is the unknown time-scale of mixing and boundary layer diffusion in the sample chamber. Because neither the flow rate nor the sample chamber geometry is changed between experiments, $k_{\mathrm{m}}$ is unchanging and can be obtained using a fitting procedure (Sec. 4).

We choose to operate in the $40 \%$ to $80 \% \mathrm{RH}$ range. In this range, the vapor diffusivity of NFC films is essentially constant, while moisture content varies almost linearly with RH (Minelli et al, 2010). 


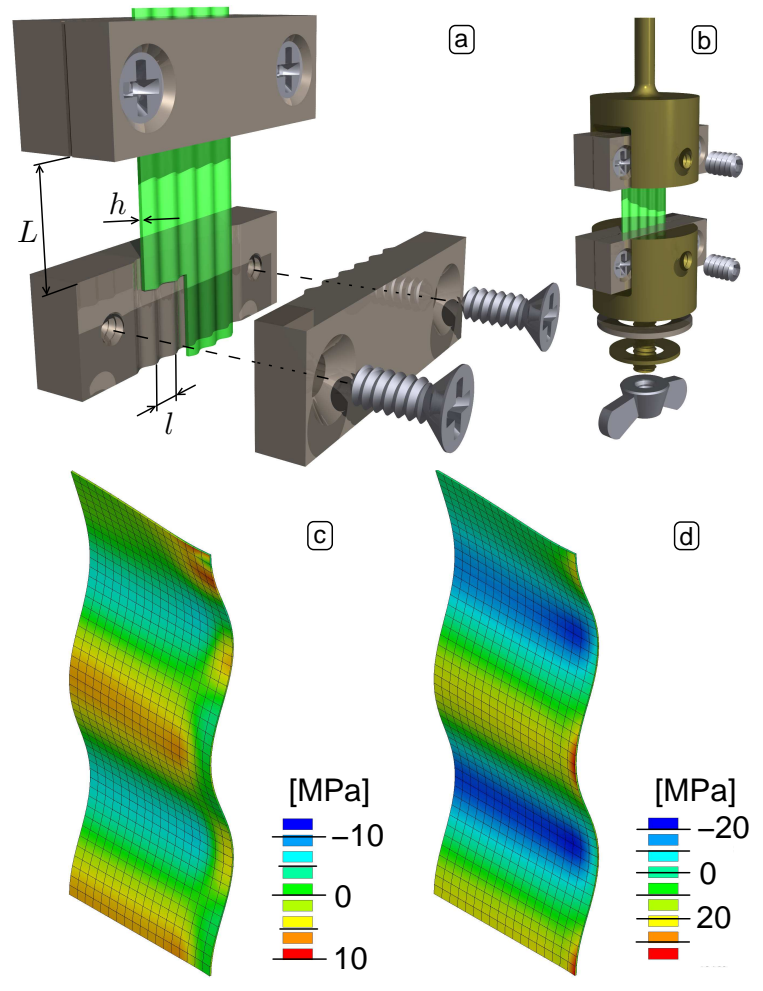

Figure 2: (a) Clamps with sinusoidal surface undulations of wavelength $l=$ $2 \mathrm{~mm}$ and amplitude $120 \mu \mathrm{m}$ are aligned and fixed together to corrugate the film, which becomes less sensitive to buckling. (b) The clamps are fixed between two holders designed for the PerkinElmer DMA7e ${ }^{\mathrm{TM}}$. (c) Surface distribution of the loading direction stress component for a quarter of the unloaded sample. A FEA prediction of elastic deformations of the F2 reference sample is shown. (d) Surface distribution of the cross direction stress component for the unloaded F2 sample. 

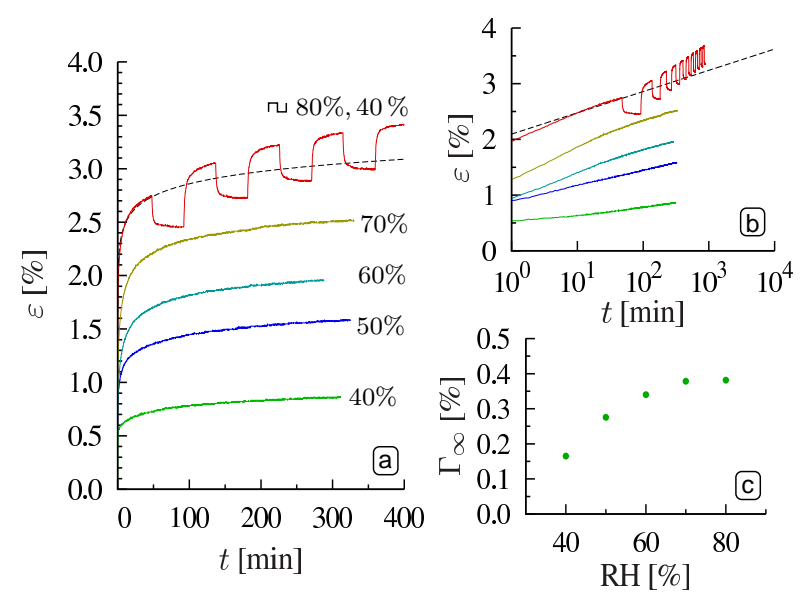

Figure 3: (a) Creep curves of the F2 NFC film for constant 40, 50, 60 and $70 \% \mathrm{RH}$, and for varying $\mathrm{RH}$ between $80 \%$ and $40 \%$ with period $90 \mathrm{~min}$. The dashed line represents a fit logarithmic function. (b) Creep curves of F2 for constant and varying $\mathrm{RH}$, respectively, in a log-lin plot. The dashed line indicates the fitted, ultimate log time creep rate at $80 \% \mathrm{RH}$. (c) Log time creep rate $\Gamma_{\infty}$ as a function of $\mathrm{RH}$.

\section{Creep and accelerated creep of NFC films}

Creep experiments are conducted in tension at $\sigma=60 \mathrm{MPa}$ for $3 \mathrm{~mm}$ long and about $6.3 \mathrm{~mm}$ wide samples. We begin by investigating the creep behavior of NFC films at constant RH. The creep strains of F2 (Table 1) for a range of constant RHs between $40 \%$ and $70 \%$ are plotted against time in a lin-lin and a log-lin plot (Figs. 3ab). After a transient of approximately 25 min duration, the strain $\epsilon$ can be approximated by a logarithmic function of time. The asymptotic creep strain is thus described by

$$
\epsilon=\Gamma_{\infty} \log _{10}(t / \tau),
$$

where $\tau$ is a time constant and $\Gamma_{\infty}$ is the log time creep rate (Wang et al, 1990), that is the amount of creep per decade of time. Note that $\Gamma_{\infty}$ is a strain; it does not have the dimensions of rate. The strongly self-delayed behavior of the creep strain is particular to tensile loading. From the empirical Eq. (2), it appears as though there is no upper limit for the creep strain. It is an outstanding question whether such a limit exists, and it should be made clear that we do not suggest that Eq. (2) can be extrapolated beyond the time-scales of our observations. Under identical measurement conditions, the observed log time creep rate varies between samples, likely because of differences in film forming conditions. For hygroscopic materials, $\Gamma_{\infty}$ typically increases with moisture concentration, as shown in Fig. 3c for F2.

The mechanosorptive creep experiments are conducted in cyclic RH be- 
tween $80 \%$ and $40 \%$, each half-cycle following the exponential plateau of Eq. (1). By considering the creep curve of $\mathrm{F} 2$ for varying $\mathrm{RH}$ with period $T=90 \mathrm{~min}$ (Figs. 3ab), the log time creep rate during $\mathrm{RH}$ cycling is found to be 2.3 times higher than that of constant $80 \% \mathrm{RH}$. The accelerated creep is thus 2.3 by definition (Wang et al, 1990). This is in agreement with some previous experimental investigations of ordinary paper (Coffin and Habeger, 2001). However, accelerated creep as high as 7.5 has also been reported (Habeger and Coffin, 2000). Assuming that the NFC film fractures at some critical strain, it is clear from Fig. 3b that the enhanced creep rate can affect the lifetime by several orders of magnitude.

The fact that mechanosorptive creep is observed for both NFC films and paper suggests that features particular to wood fibers, including a higherlevel microstructure and chemical heterogeneity, are not required for the mechanosorptive creep mechanism. This, of course, has been previously demonstrated for regenerated cellulose and some other hygroscopic polymeric materials (Habeger et al, 2001). At the same time, this does not exclude the possibility that features particular to wood fibers could contribute to the mechanosorptive creep.

The strain state of paper and NFC films depends on the drying conditions and the subsequent temperature, stress and humidity history. Hence, if no stress is applied to the material, $\sigma=0$, a relaxation of the dimensions is still observed during moisture cycling. This sorption-induced relaxation is convolved with the mechanosorptive creep response in experiments. Consequently, it becomes difficult to interpret the dynamic moisture creep behavior for small applied loads. In the present work, this problem is addressed by applying a large static loading, so that the stress-induced strain is at least comparable to the sorption-induced strain. With this strategy for isolating the mechanosorptive creep effect, the range within which stresses can be varied becomes narrow.

\section{Through-thickness moisture dynamics}

To understand the influence of the moisture dynamics on creep, we must first describe the moisture transport itself. It has previously been observed that moisture invades into nanocellulose films at two different time-scales (Minelli et al, 2010): Fickian diffusion at short time-scales and a slower moisture uptake at long time-scales which may be due to a relaxation of the cellulose matrix (Minelli et al, 2010). It should be mentioned that a similar, two time-scales sorption behavior is observed for ordinary paper, in which case vapor invades through the pore structure and diffuses into denser regions (Leisen et al, 2001; Ramarao et al, 1995), probably best described by dual transport/sorption models for vapor and condensed phase (Ramarao et al, 2003). However, the typical pore size of NFC films is in the order of 
the mean free path of water molecules in air, which is approximately $68 \mathrm{~nm}$ (Jennings, 1988). It is thus not straight-forward to identify any continuum gas phase inside the NFC film. Rather, the cellulose film can be regarded as a two-phase material comprising an inert, crystalline dispersed phase and an amorphous cellulose matrix, where density variations may exist within the latter. Thus, multiple scale moisture transport similar to that of ordinary paper may still occur within the amorphous phase of the NFC film. This would manifest as non-Fickian sorption dynamics.

We begin by investigating Fickian diffusion, which is believed to govern moisture sorption at shorter time-scales (Minelli et al, 2010). Previous experiments show that the moisture diffusivity $D$ of NFC films, while being strongly moisture-dependent at low $\mathrm{RH}$, becomes essentially constant in the $\mathrm{RH}$ range $40 \%$ to $80 \%$ investigated in this work (Minelli et al, 2010). Consider a hygroscopic film of thickness $h$. Let the $x$ coordinate axis be oriented in the thickness direction with its origin at the center of the film. Both film surfaces are in contact with air of relative humidity $H(t)$. Assuming Fickian diffusion with a constant diffusivity $D$, the moisture concentration by mass $u(x, t)$ in the film is governed by

$$
u_{t}=D u_{x x}, \quad u( \pm h / 2, t)=u_{\mathrm{eq}}[H(t)],
$$

where $u_{\text {eq }}(H)$ is the equilibrium moisture concentration. For the exponential plateau change in RH described by Eq. (1), under the assumptions that $u_{\mathrm{eq}}$ is linear in $H$ and that the film is initially in equilibrium, Eq. (3) has an analytic solution (Appendix A):

$$
\begin{aligned}
& \frac{u(x, t)-u_{0}}{u_{1}-u_{0}}= 1-\frac{\cos \sqrt{4 \alpha x^{2} / h^{2}}}{\cos \sqrt{\alpha}} e^{-k t} \\
&-2 \sum_{n=0}^{\infty}(-1)^{n} \frac{\alpha \cos \left(2 a_{n} x / h\right)}{a_{n}\left(\alpha-a_{n}^{2}\right)} e^{-a_{n}^{2} \nu t}, \\
& a_{n}=\pi(n+1 / 2), \quad \nu=4 D / h^{2}, \quad \alpha=k / \nu,
\end{aligned}
$$

where $u_{0}=u_{\text {eq }}\left(H_{0}\right)$ and $u_{1}=u_{\text {eq }}\left(H_{1}\right)$. The predicted evolution of the moisture profile is illustrated in Fig. 4a (ideal step) and Fig. $4 \mathrm{~b}(k=0.9 \nu)$, where the latter corresponds the $\mathrm{F} 2$ film and a ramp rate $k=0.16 \mathrm{~min}^{-1}$ used in some of the experiments below. Note that an ideal RH step always produces the same succession of moisture profiles, only differing by a time and length scaling, regardless of the sample thickness. Using RH ramps crucially enables changing the moisture difference between the surface and the core of the sample. An alternative method for probing the surface-core moisture difference is the swept-sine humidity cycling (Urbanik and Lee, 1995). 

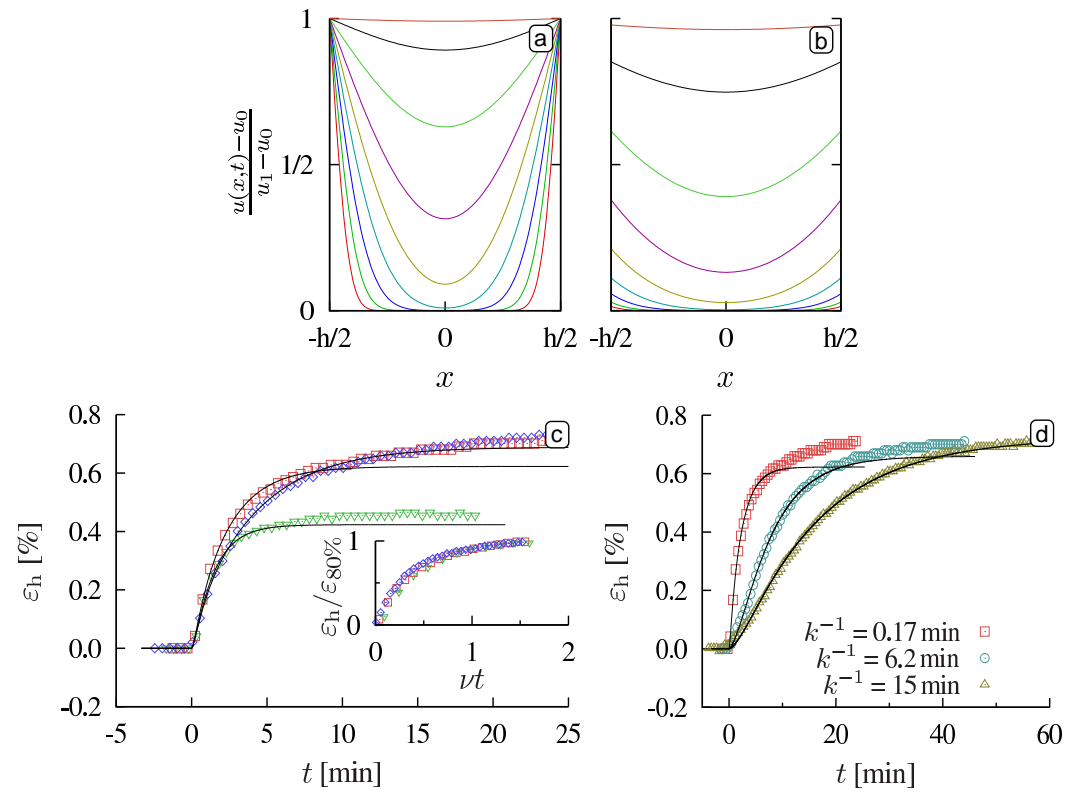

Figure 4: (a) Development of the through-thickness moisture profile, as predicted by Fickian diffusion for the ideal RH step, $k \rightarrow \infty$. The lines represent the moisture profiles at $t=2^{n} / \nu, n=-7,-6, \ldots, 1$ after the step. (b) Predicted development of the moisture profile for a finite $\mathrm{RH}$ ramp rate $k=0.9 \nu$. (c) Hygroexpansion of NFC films during a nearly ideal step from $40 \%$ to $80 \% \mathrm{RH}$ for different films: F1 (down-triangles), F2 (squares) and F3 (diamonds). Solid lines are model fits using diffusivity $D=0.16 \mu \mathrm{m}^{2} / \mathrm{s}$ and $\mathrm{RH}$ ramp rate $k=2.4 \mathrm{~min}^{-1}$ for all data sets. The inset shows that the hygroexpansion curves collapse when plotted against normalized time $\nu$, indicating Fickian diffusion. (d) Hygroexpansion of F2 with different $\mathrm{RH}$ ramp rates. Solid lines represent the model prediction with $D=0.16 \mu \mathrm{m}^{2} / \mathrm{s}$. 
Integrating Eq. (4) across the film thickness yields the average moisture concentration $\bar{u}(t)$, becoming

$$
\frac{\bar{u}(t)-u_{0}}{u_{1}-u_{0}}=1-\frac{\tan \sqrt{\alpha}}{\sqrt{\alpha}} e^{-k t}-2 \sum_{n=0}^{\infty} \frac{\alpha}{a_{n}^{2}\left(\alpha-a_{n}^{2}\right)} e^{-a_{n}^{2} \nu t} .
$$

The appearance of the rates $k$ and $\nu$ implies that there is a characteristic time-scale of sorption, which we define as $T_{\mathrm{S}}=k^{-1}+\nu^{-1}$.

We proceed to investigate Eq. (5) experimentally. After a pretreatment of at least one sorption/desorption cycle, the hygroexpansion strain $\epsilon_{\mathrm{h}}$ becomes approximately linear in $\bar{u}$ in the investigated humidity range (Uesaka et al, 1991; Strömbro and Gudmundson, 2008; Larsson et al, 2009). Thus, the hygroexpansion response follow Eq. (5) differing only by a scale factor. The samples are pretreated using two sorption cycles between $40 \%$ and $80 \%$ $\mathrm{RH}$. Then, we measure the hygroexpansion during a $\mathrm{RH}$ step from $40 \%$ to $80 \%$ for F1, F2 and F3 (Fig. 4c). Fitting the hygroexpansion equivalent of Eq. (5) to the family of data sets render the diffusivity $D=0.16 \mu \mathrm{m}^{2} / \mathrm{s}$ and the finite $\mathrm{RH}$ ramp rate $k=k_{\mathrm{m}}=2.4 \mathrm{~min}^{-1}$ due to the mixing dynamics in the sample chamber, as described by Eq. (1). The observed diffusivity is significantly lower than the previous reported $D=0.68 \mu \mathrm{m}^{2} / \mathrm{s}$ for this type of NFC film (Minelli et al, 2010), most likely due to the much higher porosity of those films (Table 1), resulting from a different drying procedure. Plotting hygroexpansion against normalized time $\nu t$ collapses the data sets at small time-scales, indicating Fickian diffusion (Fig. 4c, inset). For further validation, the hygroexpansion is measured with different $\mathrm{RH}$ ramp rates $k \in[2.4,0.16,0.065] \mathrm{min}^{-1}$, as shown in Fig. 4d. The prediction of Eq. (5) is in excellent agreement with experimental observations at short time-scales. The long time-scale sorption behavior, however, deviates from Fickian diffusion. This slow drift of the moisture content is believed to be due to a relaxation of the cellulosic matrix (Minelli et al, 2010) - the non-crystalline material between the nanocellulose crystals - and is thus not associated with any significant through-thickness moisture gradient. Therefore, it is concluded that the Fickian diffusion model, Eq. (4), very well describes the time evolution of the moisture gradient, and gives a fair prediction of the average moisture contents for the investigated range of $\mathrm{RH}$ at time-scales less than one hour.

\section{$5 \quad$ Locality of mechanosorptive creep}

To investigate whether the mechanosorptive creep is a local effect, primarily governed by local moisture changes, or a nonlocal effect, driven by macroscopic stresses induced by the through-thickness moisture profile (Habeger and Coffin, 2000), we decouple the moisture rate of change $u_{t}$ and the moisture gradient $u_{x}$ by varying the film thickness $h$ and the RH ramp rate $k$. 
The moisture difference between the surface and the core of the film is $\tilde{u}(t)=u(h / 2, t)-u(0, t)$. One possibility is that cumulated mechanosorptive creep during an $\mathrm{RH}$ ramp is governed by $\max _{t} \tilde{u}$. For ideal RH steps, the maximum of $\tilde{u}$ is essentially $\Delta u=u_{1}-u_{0}$ (Fig. 4a). Decreasing the ramp rate $k$ leads to a smaller maximum $\tilde{u}$ (Fig. $4 \mathrm{~b}$ ). That is, it would be expected that the accelerated creep decreases with $k$. Another possibility is that the mechanosorptive creep rate is controlled by $\tilde{u}$. If we take the creep rate to be linear in $\tilde{u}$, the cumulated mechanosorptive creep during one $\mathrm{RH}$ ramp should then scale with

$$
\int_{0}^{\infty} \tilde{u}(t) \mathrm{d} t=\frac{\Delta u}{2 \nu}=\frac{h^{2} \Delta u}{8 D} .
$$

Hence, the nonlocal, gradient-driven mechanosorptive creep hypothesis implies that the creep rate has a dependence on $h$ or $k$. In contrast, if the mechanosorptive creep originates from a local mechanism, as a first-order approximation, we may expect that $\mathrm{d} \epsilon \propto \mathrm{d} \bar{u}$ so that the mechanosorptive creep deformation during an $\mathrm{RH}$ step is simply a function of $\Delta u$. Consequently, the mechanosorptive creep rate should scale only weakly with $h$ or $k$, as long as $T_{\mathrm{s}} \ll T / 2$; if $T_{\mathrm{S}}$ becomes comparable to $T / 2$, the amplitude of the moisture variations would become significantly less than $\Delta u$.

Creep experiments with cyclic RH starts at time $t=0$, and at time $t_{1}=$ 40 min there is a first RH step from $80 \%$ to $40 \%$. The $\mathrm{RH}$ is then toggled between $40 \%$ and $80 \%$ with a period of $T=90 \mathrm{~min}$. To compare mechanosorptive creep curves, a normalized creep strain is defined by

$$
\hat{\epsilon}_{1}(t)=\frac{\epsilon(t)-\epsilon\left(t_{1}\right)}{\Gamma_{\infty}},
$$

which compensates for the naturally occurring variations in $\Gamma_{\infty}$. In experiments, $\Gamma_{\infty}$ is taken as the creep rate at time $t_{1}$. The normalized creep is plotted for a range of film thicknesses $h$ and $\mathrm{RH}$ ramp rates $k$, corresponding to $\nu \in[0.098,0.301] \mathrm{min}^{-1}$ and $k \in[0.16,2.4] \mathrm{min}^{-1}$, thus maintaining $T_{\mathrm{s}} \ll T / 2$. When all the normalized creep curves are plotted together (Fig. 5a), they collapse onto a single curve. The logarithmic creep rate for varying $\mathrm{RH}$ is $2.3 \Gamma_{\infty}$ with less than $\pm 8 \%$ variation due to the changes in $h$ and $k$. This shows that the mechanosorptive creep effect is independent of $h$ and $k$ within the investigated range, as long as there is a sufficient amount of time for moisture equilibration between $\mathrm{RH}$ steps. This observation is inconsistent with the gradient-driven creep hypothesis, and suggests that mechanosorptive creep is a local phenomenon in the investigated material.

When the characteristic sorption time $T_{\mathrm{S}}$ is in the order of the halfperiod $T / 2$, the amplitude of the moisture variations inside the sample is reduced (Figs. 4ab). This is known to diminish the mechanosorptive creep rate (Urbanik and Lee, 1995; Habeger et al, 2001). The effect is illustrated for NFC films by the creep curves of F2 in Fig. 5b, where the RH ramp rate 

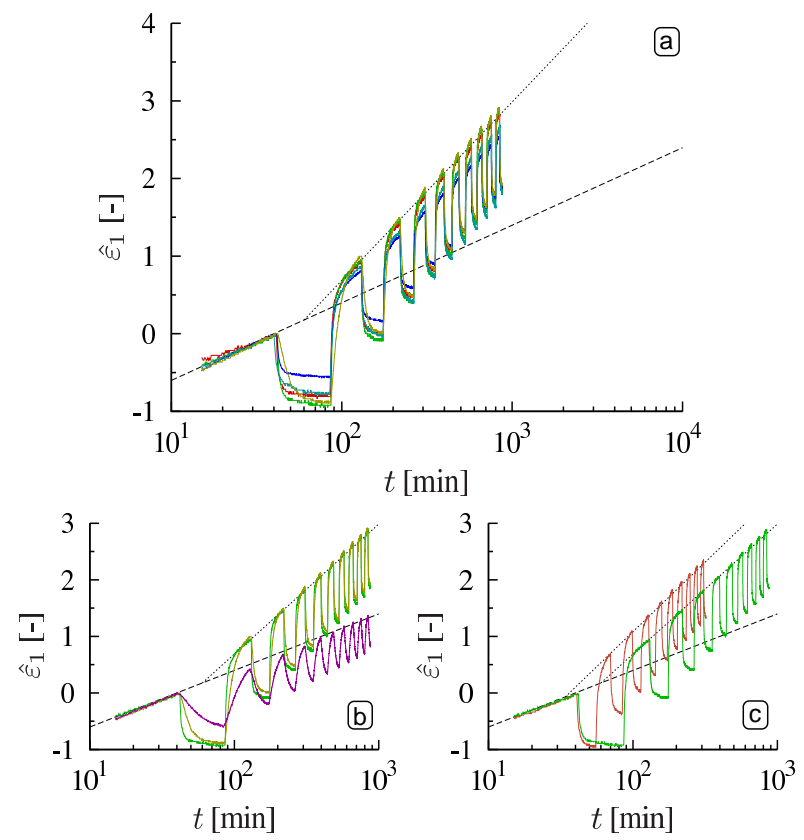

Figure 5: (a) Creep curves of NFC films with varying thickness and RH ramp rate. The ultimate $\log$ time creep rate $\Gamma_{\infty}$ at constant $80 \% \mathrm{RH}$ is indicated by the dashed line. The logarithmic creep rate for varying $\mathrm{RH}$ is $2.3 \Gamma_{\infty}$, indicated by the dotted line. (b) Creep curves of $\mathrm{F} 2$ with cyclic $\mathrm{RH}$ for different $\mathrm{RH}$ ramp rates $k \in[2.4,0.16,0.065] \mathrm{min}^{-1}$. (c) Creep curves for F2 films for different $\mathrm{RH}$ cycle periods: $T=40 \mathrm{~min}$ and $T=90 \mathrm{~min}$.

is varied: $k \in[2.4,0.16,0.065] \mathrm{min}^{-1}$. The corresponding root-mean-square of the moisture contents are $[0.47,0.41,0.30] \Delta u$, obtained by numerically solving Eq. (3) for multiple moisture cycles. Indeed, for the slowest sorption $T_{\mathrm{S}}=23$ min, only a weak mechanosorptive creep can be detected.

In the case of paper loaded in tension, it has been reported that the mechanosorptive creep is constant over each moisture cycle for a wide range of periods $20 \mathrm{~min}<T<168 \mathrm{~h}$ (Gunderson and Tobey, 1990). Thus, the accelerated creep is expected to be independent of the period as long as there is sufficient time for moisture equilibration between half-cycles; if the sorption time $T_{\mathrm{s}}$ is sufficiently short, the period $T$ of moisture variations can be reduced while still ensuring that $T_{\mathrm{S}} \ll T / 2$. The mechanosorptive creep of F2 for $T=90 \mathrm{~min}$ and $T=40 \mathrm{~min}$ and ideal $\mathrm{RH}$ ramps are compared in Fig. 5c. Reducing the period shifts the creep curve upward in a loglin plot. Even though the material clearly creeps faster, the logarithmic creep rate $\Gamma$ in tension is not significantly influenced by the period, which is consistent with observations for paper (Gunderson and Tobey, 1990). A period-dependence of the accelerated creep may exist for longer periods not 


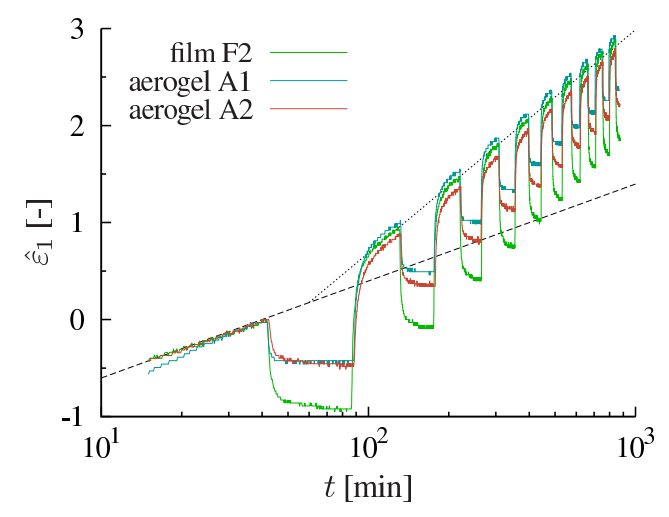

Figure 6: Comparison between mechanosorptive creep of an NFC film and NFC aerogels. RH is cycled between $40 \%$ and $80 \%$ with period $T=90 \mathrm{~min}$.

investigated in this work.

\section{Effects of microstructures in NFC materials}

While the crystalline phase of cellulosic materials is inert to moisture changes, the amorphous phase, including interfibril bonds, expands when moisture diffuses into the structure. Due to this incompatibility between the phases, moisture changes induce stress concentrations at length-scales ranging from the fibril diameter to the sample size. These stress concentrations may increase the creep rate through a mechanism reminiscent of heterogeneitydriven creep (Habeger and Coffin, 2000). By comparing the creep behavior of nanocellulose materials with very different microstructures, it would be possible to identify the relevant length-scales at which the governing mechanism of mechanosorptive creep is active. Thus, we compare the creep of NFC films with the creep behavior of NFC aerogels. The high-porosity aerogels (Fig. 1b) have more forgiving microstructures than the films (Fig. 1a); the hygroexpansion strains and stresses are localized to the bond sites, and the relatively long unbonded fibril segments preclude any stress-enhancing, cooperative effect over length-scales larger than the fibril diameter.

The normalized creep curves of F2, A1 and A2 are compared in Fig. 6 for $T=90 \mathrm{~min}$ and ideal step $\mathrm{RH}$ variations between $40 \%$ and $80 \%$. Samples $\mathrm{A} 1$ and $\mathrm{A} 2$ are tested in an uncorrugated geometry, using tensile stresses $\sigma=$ 2.0 and $\sigma=4.1 \mathrm{MPa}$, respectively, to produce the same specific stress $\sigma / \rho \approx$ $12 \mathrm{kNm} / \mathrm{kg}$ for both samples. The specific stress applied to F2 is higher, $\sigma / \rho \approx 41 \mathrm{kNm} / \mathrm{kg}$, due to experimental limitations. Still, since the accelerated creep is not very sensitive to stress (Habeger and Coffin, 2000), the comparison between films and aerogels is relevant. The diffusivities for A1 and A2 are $D=43 \mu \mathrm{m}^{2} / \mathrm{s}$ and $D=15 \mu \mathrm{m}^{2} / \mathrm{s}$, as estimated from their hygroexpansion dynamics. This ensures moisture equilibration be- 
tween half-cycles. The aerogels exhibit a significant mechanosorptive creep. Remarkably, the films and the aerogels show the same accelerated creep, 2.3 with less than $\pm 5 \%$ variation, although they have completely different microstructures. This is consistent with mechanosorptive creep mechanisms occurring at the length-scales of the fibril diameter down to molecular scales. In contrast, any heterogeneity-induced stresses at length-scales greater than the fibril diameter should be very different in films and aerogels, demonstrating that the mesostructure stress variations are less important for the mechanosorptive creep of NFC-based materials.

The present experiments indicate that levels of structure larger than the fibril diameter are of limited importance to the development of accelerated creep when the configuration of fibrils is random, as is the case in the NFC materials studied above. Previous investigations of paper and board show that higher levels of structure can have some amplifying effect on the mechanosorptive creep rate. For instance, using pulps of different moisture sensitivity in different plies within the paper sheet has been observed to increase the accelerated creep by about 13\% (Coffin and Habeger, 2001). However, for the primary mechanosorptive creep mechanism, a much stronger effect would be expected. In the case of nanocellulose materials, it is still possible that the heterogeneity-driven creep is dominant at the length-scale of the fibril diameter. On the other hand, designing an experiment to prove this is obviously difficult due to the small length-scales. Another plausible hypothesis, not tested herein, is that the mechanosorptive creep is an effect of physical de-aging due to a drying cycle in conjunction with physical aging of the material (Padanyi, 1993). It has been shown experimentally for paper that a drying cycle effectively rejuvenates the material, resulting in a strong increase in creep rate (Habeger and Coffin, 2000). Since physical aging, together with temperature, is the most important parameter determining the creep compliance of glassy polymers (Struik, 1977), additional experimental investigations of the effect of aging on the accelerated creep of nanocellulose materials would be of great interest.

\section{Conclusions}

Since mechanosorptive creep is observed in NFC films and aerogels, it is concluded that microstructures particular to naturally occurring fiber agglomerates, such as microfibrils or wood fibers, are not necessary for the phenomenon to occur in NFC-based materials. Neither is anisotropic swelling at the fiber level required. The possibility that such mesostructure effects could enhance mechanosorptive creep can, however, not be excluded.

As long as there is a sufficient amount of time for moisture equilibration during each step of the $\mathrm{RH}$ cycle, the mechanosorptive log time creep rate does not significantly depend on $\mathrm{RH}$ ramp rate or sample thickness. These 
observations indicate that mechanosorptive creep is a local phenomenon, while the effect of spatial moisture variations at the scale of the sample thickness is negligible. Moreover, since NFC aerogels show as much accelerated creep as do NFC films, there is no evidence that any structural length-scale larger than the fibril diameter contributes to mechanosorptive creep. This suggests that mechanosorptive creep in NFC-based materials should be attributed mainly to the interfibril bonds or possibly to the fibrils themselves.

\section{Acknowledgements}

Anne-Mari Olsson and Lennart Salmén, Innventia AB, are acknowledged for their assistance during the experiments. S.B.L. thanks BiMaC Innovation for financial support.

\section{A Fickian diffusion with exponential plateau bound- ary conditions}

Symmetric one-dimensional diffusion of moisture in a film with exponential plateau Dirichlet boundary conditions is solved in nondimensional form for brevity. Taking the initial state to be equilibrium and using the symmetry around the center of the film render

$$
\begin{aligned}
u_{t} & =u_{x x} \\
u_{x}(0, t) & =0 \\
u(1, t) & =1-e^{-\alpha t} \\
u(x, 0) & =0,
\end{aligned}
$$

where $x \in[0,1]$ and $\alpha$ is a constant. Taking the Laplace transform gives

$$
\begin{aligned}
s U-u(x, 0) & =U_{x x} \\
U_{x}(0, s) & =0 \\
U(1, s) & =\frac{1}{s}-\frac{1}{s+\alpha},
\end{aligned}
$$

where $U(s)=\mathcal{L}[u(t)]$. The solution of Eq. (9a) is readily obtained as

$$
U(x, s)=A(s) e^{x \sqrt{s}}+B(s) e^{-x \sqrt{s}} .
$$

Equation (10) and the boundary conditions (9b) and (9c) yield

$$
U(x, s)=\frac{\cosh \sqrt{s x^{2}}}{\cosh \sqrt{s}} \cdot \frac{\alpha}{s(s+\alpha)} .
$$


Direct application of the Laplace transform inversion formula renders

$$
u(x, t)=\lim _{b \rightarrow \infty} \frac{1}{2 \pi i} \int_{a-b i}^{a+b i} e^{s t} U(x, s) \mathrm{d} s, \quad a>0 .
$$

Since the integrand is analytic in the complex plane, except at the poles $s=$ $0, s=-\alpha$ and $s=-\pi^{2}(n+1 / 2)^{2}, n=0,1, \ldots$, and since the integral vanishes when $|s| \rightarrow \infty, \Re(s)<0$, we may compute $u(x, t)$ as the sum of residues

$$
\begin{aligned}
u(x, t)= & \operatorname{Res}\left[e^{s t} U(x, s), 0\right]+\operatorname{Res}\left[e^{s t} U(x, s),-\alpha\right]+ \\
& \sum_{n=0}^{\infty} \operatorname{Res}\left[e^{s t} U(x, s),-\pi^{2}(n+1 / 2)^{2}\right] \\
= & 1-\frac{\cos \sqrt{\alpha x^{2}}}{\cos \sqrt{\alpha}} e^{-\alpha t}-2 \sum_{n=0}^{\infty}(-1)^{n} \frac{\alpha \cos \left(a_{n} x\right)}{a_{n}\left(\alpha-a_{n}^{2}\right)} e^{-a_{n}^{2} t},
\end{aligned}
$$

where $a_{n}=\pi(n+1 / 2)$. Note that the equations in this appendix are all in nondimensional form.

\section{References}

Alfthan J, Gudmundson P (2005) Linear constitutive model for mechanosorptive creep in paper. Int J Solids Struct 42:6261-6276

Armstrong LD, Christensen GN (1961) Influence of moisture changes on deformation of wood under stress. Nature 191(4791):869-870

Armstrong LD, Kingston RST (1960) Effect of moisture changes on creep in wood. Nature 185(4716):862-863

Aulin C, Netrval J, Wågberg L, Lindström T (2010) Aerogels from nanofibrillated cellulose with tunable oleophobicity. Soft Matter 6:3298-3305

Byrd VL (1972) Effects of relative humidity changes during creep on handsheet paper properties. Tappi J 55(2):247-252

Capadona JR, Shanmuganathan K, Triftschuh S, Seidel S, Rowan SJ, Weder C (2009) Polymer nanocomposites with nanowhiskers isolated from microcrystalline cellulose. Biomacromolecules 10(4):712-716

Coffin DW, Habeger CC (2001) The practical influence of heterogeneity on tensile accelerated creep in paper. Tappi J 84(3):59-59

Fahlén J, Salmén L (2002) On the lamellar structure of the tracheid cell wall. Plant Biology 4(3):339-345 
Fall AB, Lindström SB, Sundman O, Ödberg L, Wågberg L (2011) Colloidal stability of nanofibrillated cellulose dispersions. Langmuir 27(18):11,33211,338

Forgacs OL, Mason SG (1958) The flexibility of wood-pulp fibers. Tappi J 41:695-704

Gibson EJ (1965) Creep of wood: Role of water and effect of a changing moisture content. Nature 206(4980):213-215

Gunderson DE, Tobey WE (1990) Tensile creep of paperboard - effect of humidity change rates. In: Materials interactions relevant to the pulp, paper and wood industries, Mater. Res. Soc., Pittsburg, USA, San Fransisco, USA., pp 213-226

Habeger CC, Coffin DW (2000) The role of stress concentrations in accelerated creep and sorption-induced physical aging. J Pulp Paper Sci $26: 145-157$

Habeger CC, Coffin DW, Hojjatie B (2001) Influence of humidity cycling parameters on the moisture-accelerated creep of polymeric fibers. J Polym Sci B Polym Phys 39:2048-2062

Haslach HW (1994) The mechanics of moisture accelerated tensile creep in paper. Tappi J 77(10):179-186

Henriksson M, Henriksson G, Berglund LA, Lindström T (2007) An environmentally friendly method for enzyme-assisted preparation of microfibrillated cellulose (MFC) nanofibers. Eur Polym J 43(8):3434-3441

Henriksson M, Berglund LA, Isaksson P, Lindström T, Nishino T (2008) Cellulose nanopaper structures of high toughness. Biomacromolecules 9(6):1579-1585

Jennings SG (1988) The mean free path in air. J Aerosol Sci 19(2):159-166

Larsson PA, Hoc M, Wågberg L (2009) The influence of grammage. moisture content, fibre furnish, and chemical modifications on the hygro- and hydroexpansion of paper. In: 14th Fund. Res. Symp., Oxford, UK, vol 1, pp $355-388$

Leake CH, Wojcik R (1993) Humidity cycling rates: how they influence the container life spans. Tappi 76(10):26-30

Leisen J, Hojjatie B, Coffin DW, Beckham HW (2001) In-plane moisture transport in paper detected by magnetic resonance imaging. Ind Eng Chem Res 19(1):199-206 
Mackay BH, Dowes JG (1959) The effects of the sorption process on the dynamic rigidity modulus of the wool fibers. J Appl Polym Sci 2(4):32-38

Minelli M, Baschetti MG, Doghieri F, Ankerfors M, Lindström T, Siróc I, Plackett D (2010) Investigation of mass transport properties of microfibrillated cellulose (MFC) films. J Membrane Sci 358:67-75

Navi P, Pittet V, Plummer CJG (2002) Transient moisture effects on wood creep. Wood Sci Tech 36:447-462

Olsson AM, Salmén L, Eder M, Burgert I (2007) Mechano-sorptive creep in wood fibres. Wood Sci Technol 41:59-67

Padanyi ZV (1993) Physical aging and the glass transition: Effects on the mechanical properties of paper and board. In: 10th Fund. Res. Symp., Oxford, UK, vol 1, pp 521-545

Ramarao BV, Chatterjee SG, Tien C (1995) Moisture transport and sorption by paper under cyclic variations in humidity. In: Perkins R (ed) Mechanics of cellulosic materials, vol AMD-Vol. 209/MD-Vol. 60, ASME, pp 195-214

Ramarao BV, Massoquete A, Lavrykov S, Ramaswamy S (2003) Moisture transport paper materials in the hygroscopic range and characteristics of diffusion parameters. Drying Tech 21(10):2007-2056

Ranta-Maunus A (1975) Viscoelasticity of wood at varying moisture-content. Wood Sci Technol 9(3):189-205

Saito T, Kimura S, Nishiyama Y, Isogai A (2007) Cellulose nanofibers prepared by TEMPO-mediated oxidation of native cellulose. Biomacromolecules 8(8):2485-2491

Sehaqui H, Zhou Q, Ikalla O, Berglund LA (2011) Strong and tough cellulose nanopaper with high specific surface area and porosity. Biomacromolecules 12(10):3638-3644

Söremark C, Fellers CN (1989) Mechano-sorptive creep and hygroexpansion of corrugated board in bending. J Pulp Paper Sci 19(1):J19-J26

Söremark C, Fellers CN, Henriksson L (1993) Mechano-sorptive creep of paper: Influence of drying restraint and fibre orientation. In: 10th Fund. Res. Symp., Oxford, UK, vol 1, pp 547-574

Strömbro J, Gudmundson P (2008) An anisotropic fibre-network model for mechano-sorptive creep in paper. Int J Solids Struct 45:5765-5787

Struik LCE (1977) Physical aging in plastics and other glassy materials. Polym Eng Sci 17(3):165-173 
Sugiyama J, Vuong R, Chanzy H (1991) Electron diffraction study on the two crystalline phases occurring in native cellulose from an algal cell wall. Macromolecules 24(14):4168-4175

Uesaka T, Moss C, Nanri Y (1991) Characterization of hygroexpansivity of paper. In: International Paper Physics Conference Proceedings, Kona, HI, pp 613-622

Urbanik TJ (1995) Hygroexpansion-creep model for corrugated fiberboard. Wood Fiber Sci 27(2):134-140

Urbanik TJ, Lee SK (1995) Swept sine humidity schedule for testing cyclic period effects on creep. Wood Fiber Sci 27(1):68-78

Wågberg L, Annergren GO (1997) Physicochemical characterization of papermaking fibers. In: Baker CF (ed) 11th Fund. Res. Symp., vol 1, Cambridge, UK, pp 1-82

Wang JZ, Dillard DA, Wolcott MP, Kamke FA, Wilkes GL (1990) Transient moisture effects in fibers and composite-materials. J Compos Mater 24(9):994-1009

Yousefi H, Nishino T, Faezipour M, Ebrahimi G, Shakeri A (2011) Direct fabrication of all-cellulose nanocomposite from cellulose microfibers using ionic liquid-based nanowelding. Biomacromolecules 12(11):4080-4085 